Spatiotemporal Temperature and Pressure in Thermoplasmonic Gold Nanosphere-Water Systems

Sarah A. Lindley, ${ }^{1,+}$ Qi An, ${ }^{2,+}$ William A. Goddard III, 3,* Jason K. Cooper, ${ }^{1, *}$

${ }^{1}$ Chemical Sciences Division, Joint Center for Artificial Photosynthesis, Lawrence Berkeley National Laboratory, Berkeley, CA 94720, USA

${ }^{2}$ Department of Chemical and Materials Engineering, University of Nevada, Reno, NV 89577, USA

${ }^{3}$ Materials and Procs Simulation Center (MSC), California Institute of Technology, Pasadena, CA 91125, USA

*Corresponding: jkcooper@lbl.gov, wag@caltech.edu

$\dagger$, these authors contributed equally

KEYWORDS: thermoplasmonics, transient absorption spectroscopy, molecular dynamics, hollow gold nanospheres, surface plasmon resonance, heat transfer

Supporting Information 

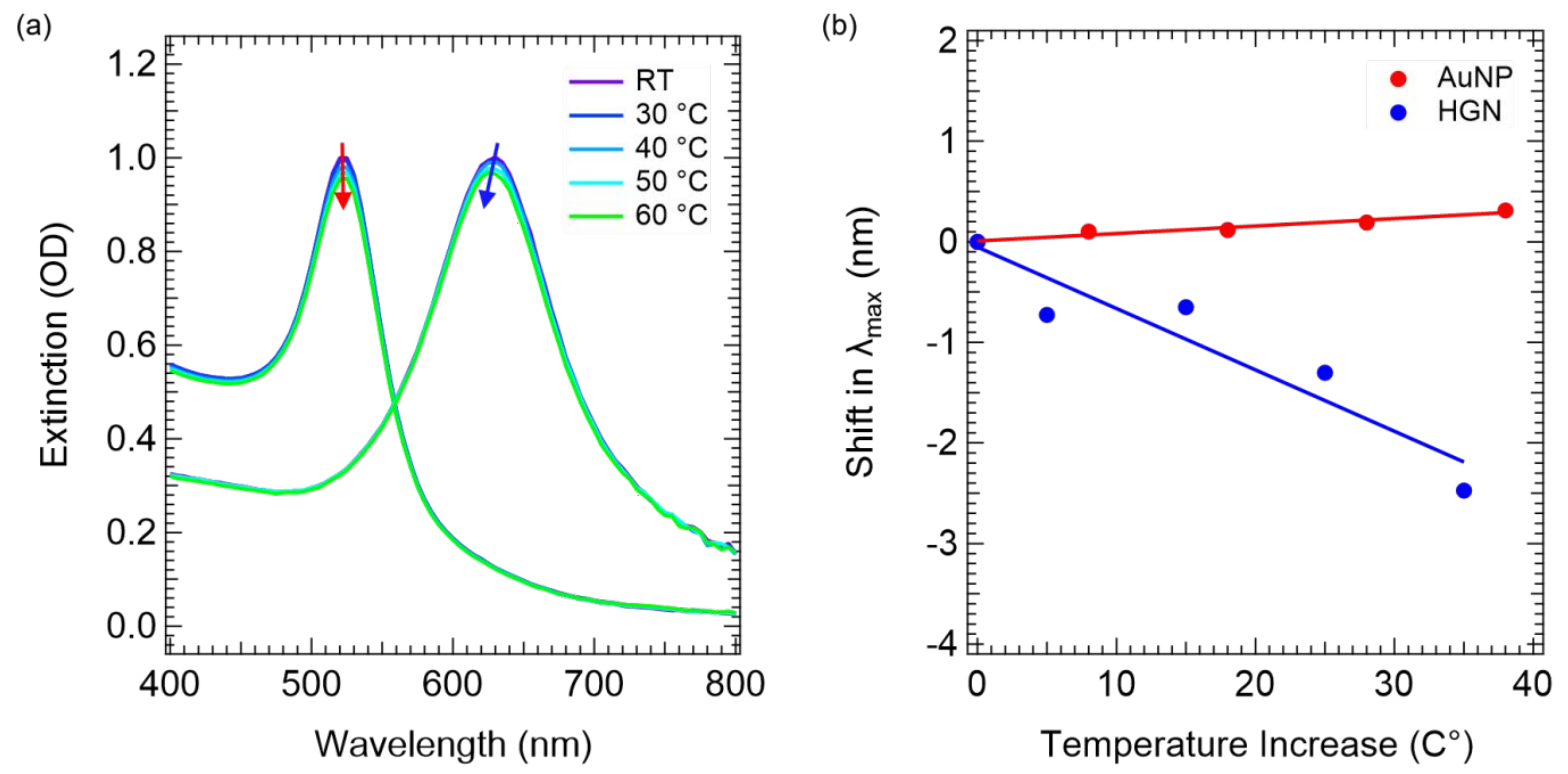

Figure S1. Temperature dependence of surface plasmon resonance (SPR) for AuNPs and HGNs. (a) Extinction for (left) and HGN (right) at temperatures ranging from room temperature (RT) to $60{ }^{\circ} \mathrm{C}$. The shift in peak SPR wavelength $\left(\lambda_{\max }\right)$ with increasing temperature is highlighted with an arrow (red arrow for AuNPs and blue arrow for HGNs). (b) The shift in surface plasmon resonance peak wavelength plotted as a function of temperature increase. In each case, $\lambda_{\max }$ was determined by taking the first derivative of the extinction and locating the wavelength corresponding to 0 OD. Linear fits are provided (solid lines), with slopes of $0.007 \pm 0.001 \mathrm{~nm} / \mathrm{C}^{\circ}$ for AuNP and $-0.06 \pm$ $0.01 \mathrm{~nm} / \mathrm{C}^{\circ}$ for $\mathrm{HGN}$. Thus, the $\mathrm{HGN}$ has nearly a 10 -fold increase in thermal-optical sensitivity as compared to the AuNP. It should be noted that the 10 -fold increase is specific to the structures analyzed within this study; we expect the sensitivity improvement to be dependent upon particle diameter and shell thickness, with greater sensitivity enhancement accessible with larger particles and thinner shells. 

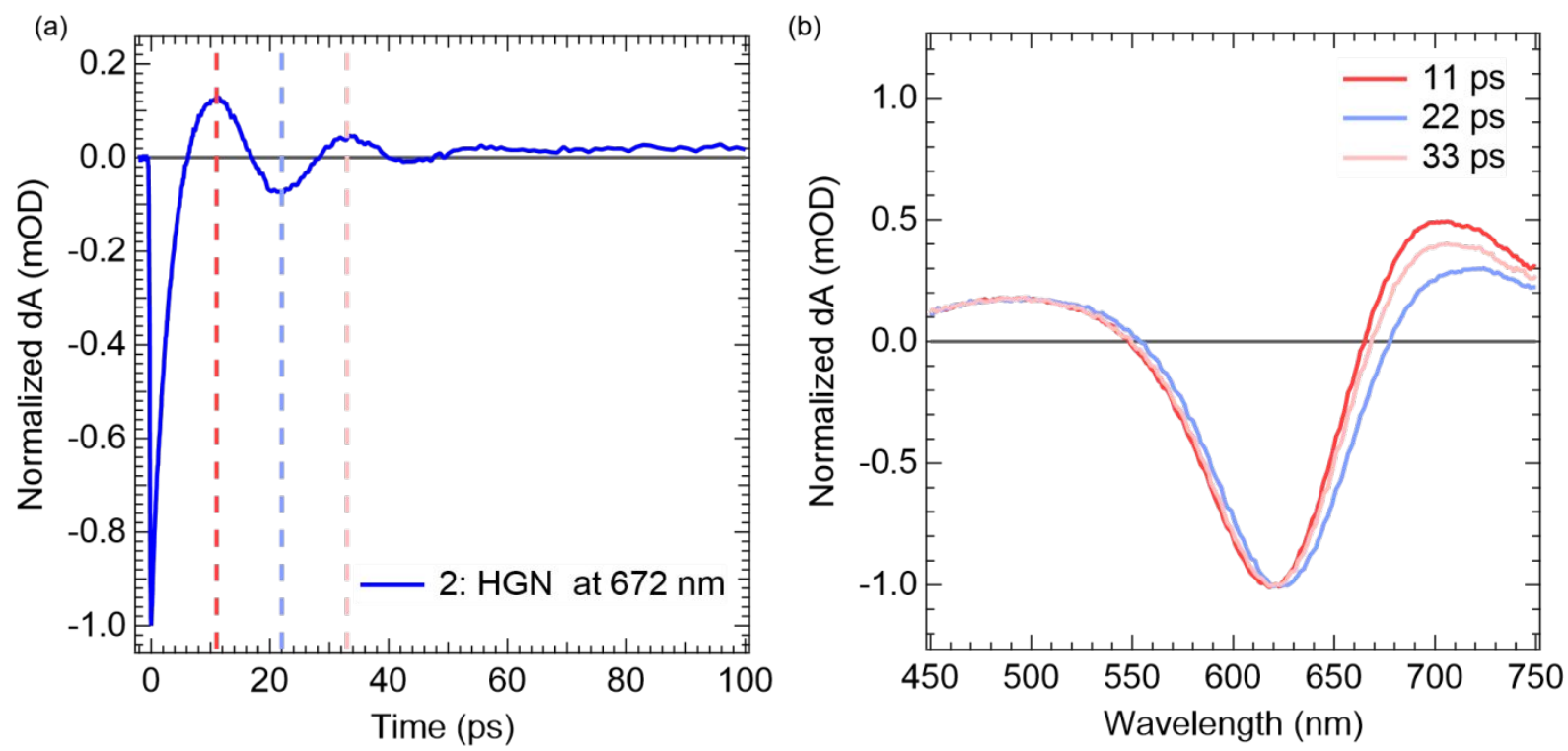

Figure S2. Transient absorption spectroscopy for HGN sample (350 nm, 500 nJ, 130 fs excitation) showing (a) time-resolved charge carrier relaxation dynamics for the $672 \mathrm{~nm}$ probe with (b) difference absorption (dA) spectral profile at select time points after excitation. Time points of 11 ps, 22 ps, and 33 ps correspond to the first peak, first trough, and second peak in the dA oscillations observed with the $672 \mathrm{~nm}$ probe wavelength. The peaks and troughs of the oscillations at this probe wavelength mainly correspond with expansion on the right side of the SPR profile (longer wavelengths) which may be correlated with thermal expansion and contraction of the lattice. As the lattice expands (cools) from 11 ps to 22 ps, the SPR becomes broader and gains signal at longer wavelengths. As the lattice contracts (heats) from 22 ps to 33 ps, the SPR shrinks back, losing signal at the longer wavelengths. Note that these time points do not directly correlate with the expansion and contraction time points for MD because MD time scales were started after particle excitation whereas TA time scales include particle excitation. Here, the 11 ps TA time point represents the heated particle and may be correlated with the 0 ps MD time point in the main manuscript. 
(a)

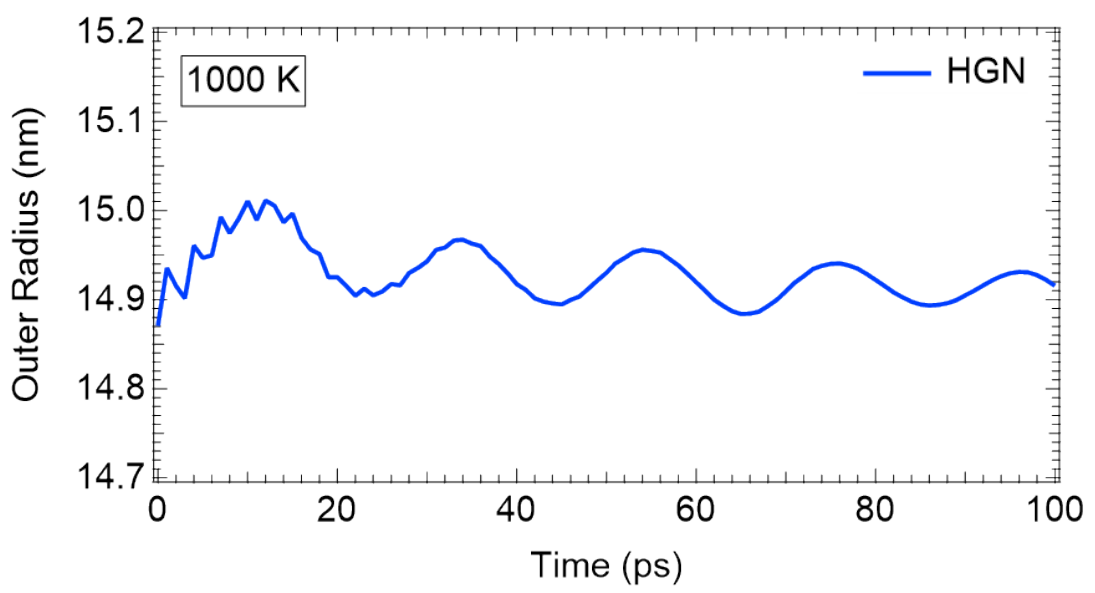

(b)

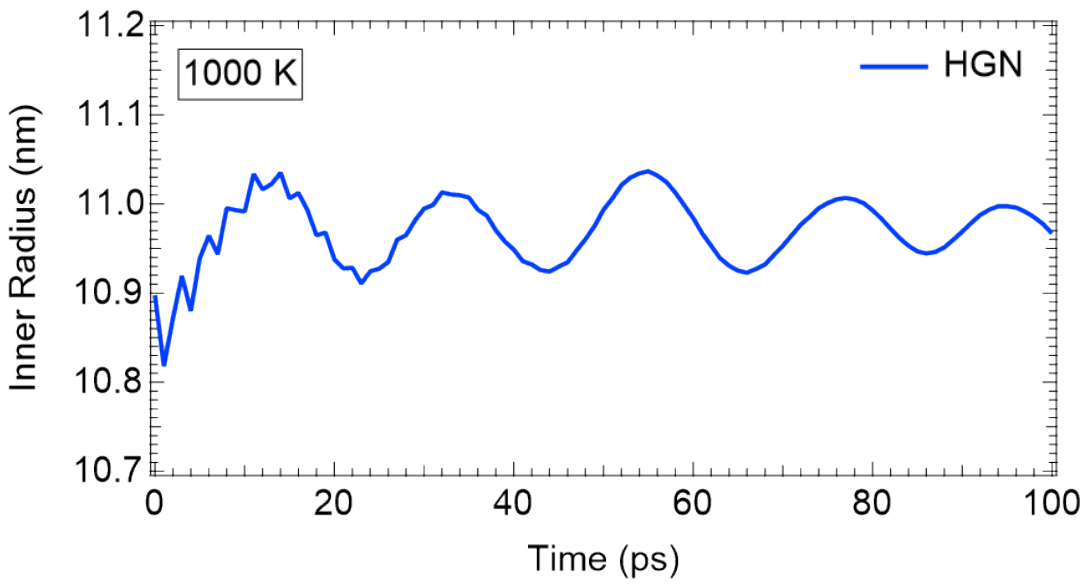

(c)

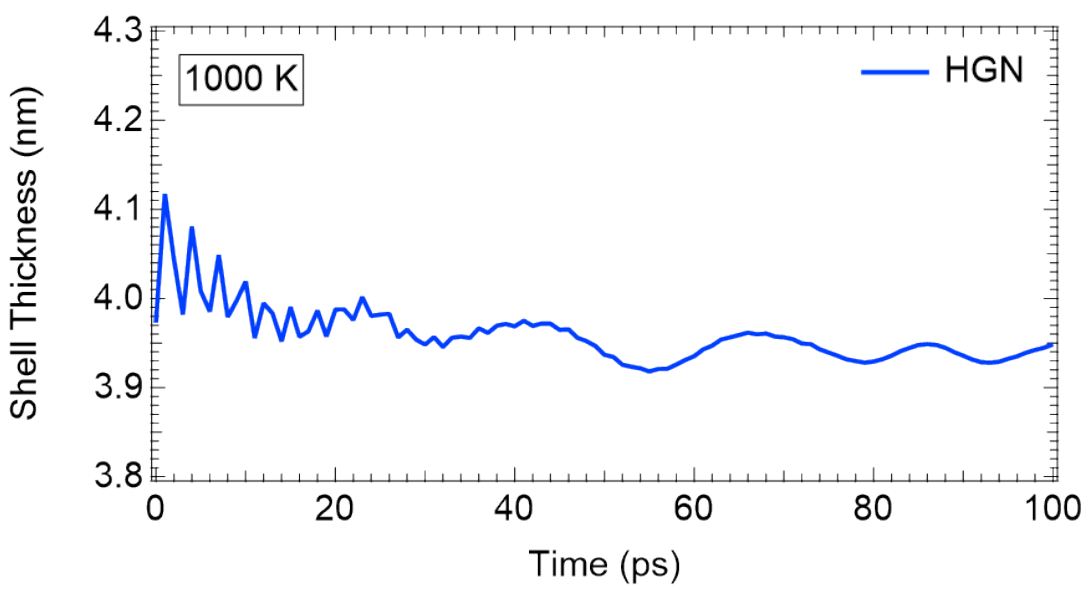

Figure S3. Thermal expansion and contraction of HGN (a) outer radius, (b) inner radius, and (c) shell thickness from 0-100 ps after ultrafast lattice heating to $1000 \mathrm{~K}$. Data extracted from molecular dynamics simulation of HGN-water system with regular lattice. 


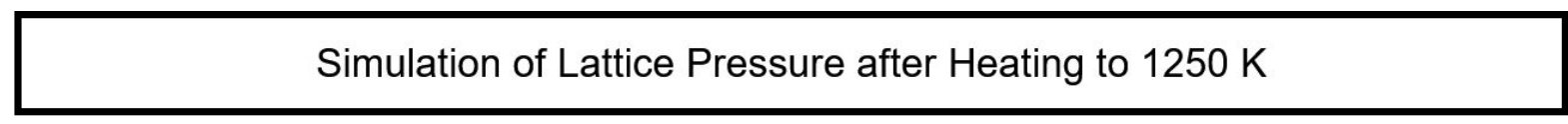

\section{Particle Radius}

(a)

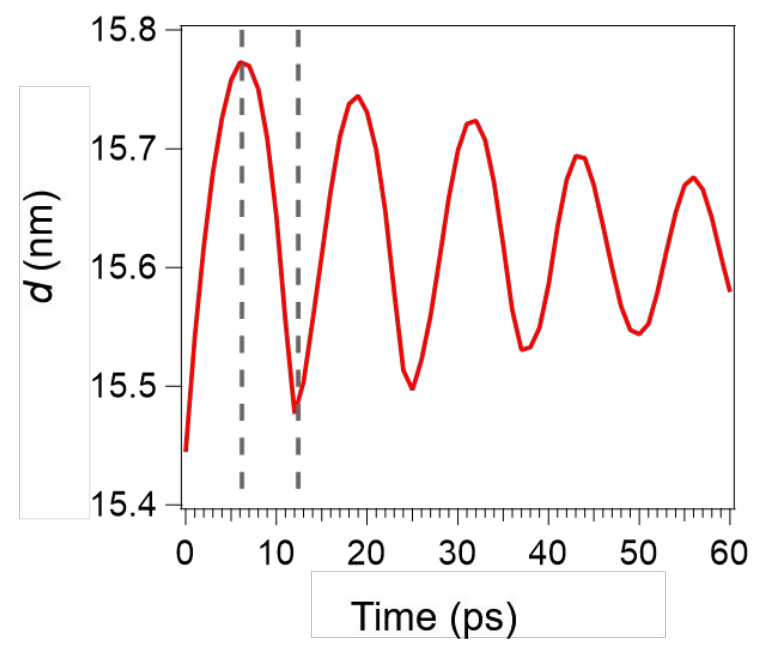

\section{Lattice Pressure (GPa)}

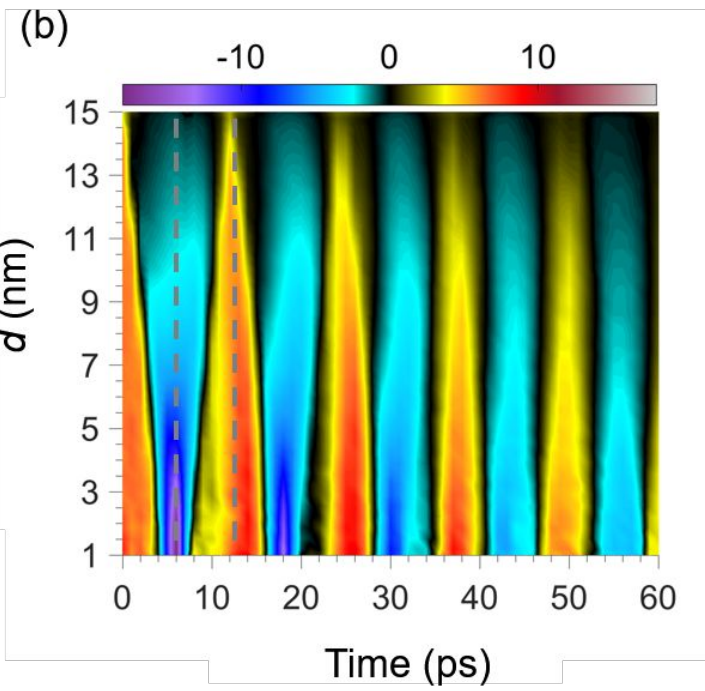

Figure S4. (a) Thermal expansion and contraction of the particle radius, with (b) spatiotemporal evolution of lattice temperature for the AuNP over 0-60 ps after lattice heating to $1250 \mathrm{~K}$. Data extracted from molecular dynamics simulation of AuNP-water system. Two dashed lines are provided on each plot to indicate timepoints corresponding to initial expansion (first line, $6.2 \mathrm{ps)}$ and contraction (second line, $12.4 \mathrm{ps}$ ). 
Simulation of Lattice Temperature after Heating to $1250 \mathrm{~K}$

\section{Shell Thickness}

(a)

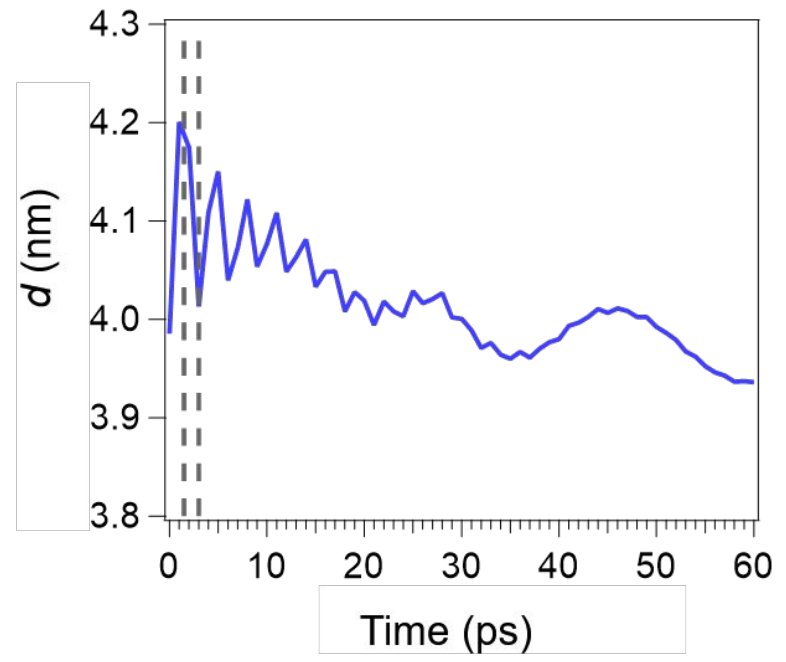

Lattice Temperature (K)

(b) $300 \quad 650 \quad 1000 \quad 1350 \quad 1700$

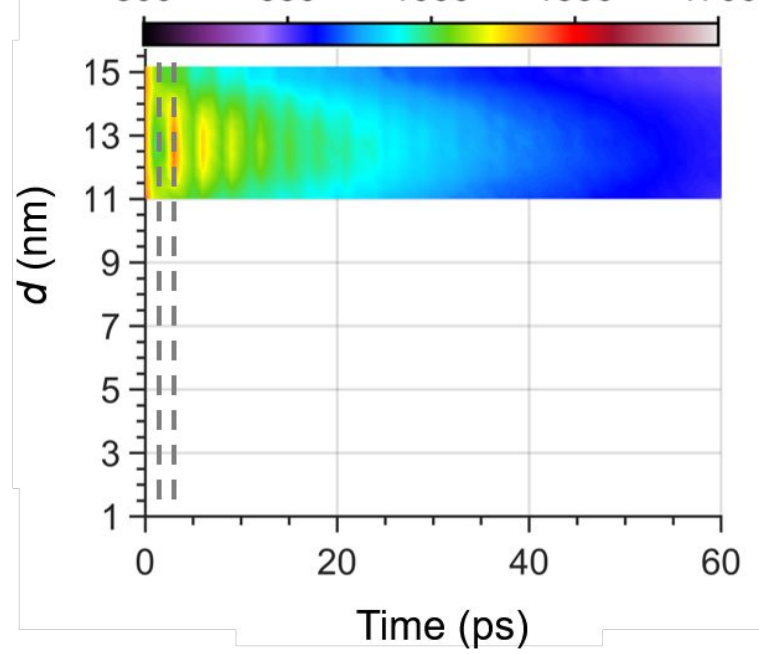

Figure S5. Comparison of shell thickness and temperature oscillations for the HGN over 0-60 ps after lattice heating to $1250 \mathrm{~K}$. An increase in shell thickness corresponds to a decrease in lattice temperature. Data extracted from molecular dynamics simulation of $\mathrm{HGN}$-water system. Two dashed lines are provided on each plot to indicate timepoints corresponding to initial shell expansion (first line, $1.5 \mathrm{ps}$ ) and contraction (second line, $3 \mathrm{ps}$ ). 
Analytical estimate of average lattice temperature oscillations using the Grüneisen equation of state:

An analytical estimate can be provided by using an equation of state for a crystalline system that relates $\mathrm{T}$ and $\mathrm{V}$. The Grüneisen equation of state describes how changes in volume of a crystal lattice affect its vibrational properties, one formulation of which is given in Equation S1,

$$
\boldsymbol{\Gamma}=\frac{\alpha v_{s}^{?}}{C_{P}}=-\left(\frac{\partial \ln T}{\partial \ln V}\right)_{S}=-\frac{V}{T}\left(\frac{\partial T}{\partial V}\right)_{S}
$$

where $\boldsymbol{\Gamma}$ is Grüneisen parameter, $\alpha$ is the volume thermal expansion coefficient, $v_{s}$ is the speed of sound in the medium, and $C_{P}$ is the principal heat capacity at constant pressure. This relationship states that when you compress a material isentropically, its temperature increases.

Rearranging Equation $\mathbf{S 1}$ and solving for the derivative for $\mathrm{T}_{2}$ puts the relationship into a form that is useful for assessing temperature change,

$$
\begin{gathered}
\frac{\partial T}{T}=-\Gamma\left(\frac{\partial V}{V}\right) \\
\ln \left(\frac{T_{1}}{T_{2}}\right)=-\Gamma \ln \left(\frac{V_{1}}{V_{2}}\right) \\
T_{2}=\frac{T_{1}}{e^{\left(-\Gamma \ln \left(\frac{V_{1}}{V_{2}}\right)\right)}}
\end{gathered}
$$

The temperature of the compressed lattice may be determined using the initial temperature, initial and final volume, and Grüneisen parameter. We will use the first compression cycle for our estimations. It should be noted that $\boldsymbol{\Gamma}$ may be computed directly from standard values for $\alpha, v_{S}$, and $C_{P}$. However, these values vary with temperature and pressure. ${ }^{1,2}$ Multiple sources have reported a $\Gamma$ value $\sim 3$ for gold $\sim 1000 \mathrm{~K}, 3,4$ which we have used in our calculations.

Expansion and contraction of the lattice after thermal excitation to $1000 \mathrm{~K}$ is provided in Figure S6 for both AuNP and HGN structures. These data were generated through MD simulation and sampled at 1 ps intervals. For each structure, the first compression cycle is noted, where $r_{1}$ represents the data point closest to the expanded radius and $r_{2}$ represents the data point closest to the compressed radius. These dimensions are reported in Table $\mathbf{S 1}$ along with corresponding lattice volumes. The change in radius is noted in Figure $\mathbf{S 6}$ for the AuNP radius, the HGN outer 
radius, and the HGN inner radius. For the HGN, we analyze the low frequency oscillation (whole particle compression) as well as the high frequency oscillation (shell wall compression).
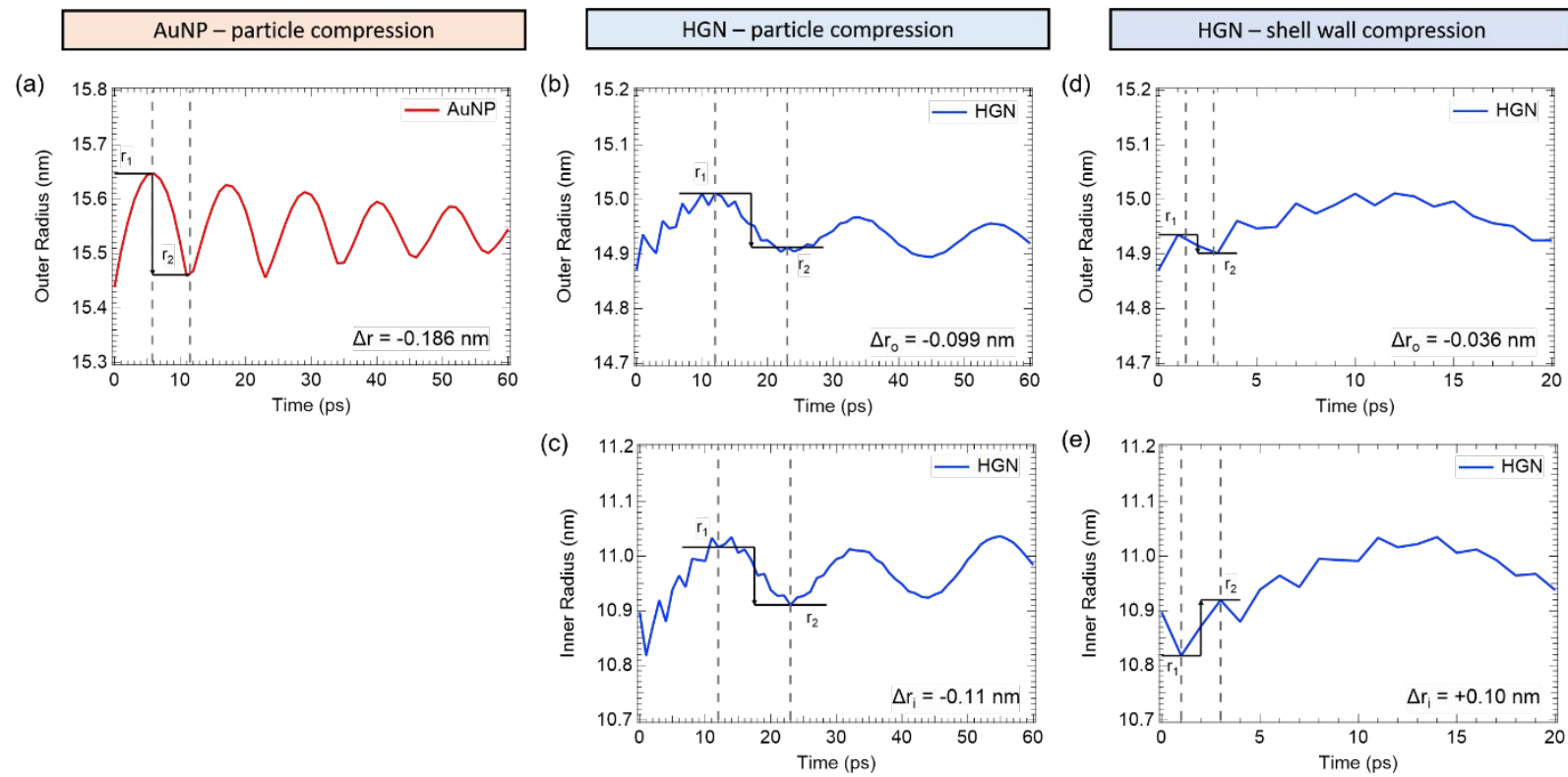

Figure S6. MD Simulation of Lattice Dimensions. Expansion and contraction of (a) AuNP outer radius, (b) HGN outer radius and (c) HGN inner radius from 0-60 ps after ultrafast heating of the lattice to $1000 \mathrm{~K}$. Expansion and contraction of (h) HGN outer radius and (i) inner radius from 020 ps is also provided to highlight the high frequency oscillation of the HGN shell wall at early times. In each case, dashed lines are provided to note the time points closest to the first observable compression: 6 ps and 12 ps for AuNP, 12 ps and 23 ps for HGN (low frequency oscillation), and 1 ps and 3 ps for the HGN shell wall (high frequency oscillation).

Using the lattice volume values in Equation S2, we estimate the temperature of the compressed AuNP to be $961 \mathrm{~K}$ (representing a temperature increase of $95 \mathrm{~K}$ ), the temperature of the compressed $\mathrm{HGN}$ to be $838 \mathrm{~K}$ (representing a temperature increase of $34 \mathrm{~K}$ ), and the temperature of the HGN with compressed shell wall to be $966 \mathrm{~K}$ (representing a temperature increase of 86.9 $\mathrm{K})$. These results are also included in Table S1.

With analytical estimates in hand, we may now review the results from our MD simulation. It should be noted that since heat from the gold lattice is constantly being transferred to the surrounding water in our MD system, we are not in an isentropic regime. As such, we expect that 
our MD simulations may show a smaller rise in temperature, particularly for the HGN which has faster heat transfer to the surrounding water due to the increased interfacial area.

MD simulation of lattice temperature after heating to $1000 \mathrm{~K}$ is provided in Figure S7. The AuNP and HGN lattice temperatures oscillate slightly as they cool. The oscillations are more visible when the lattice temperature is fit with a double exponential decay (to account for the heat dissipation to the surrounding water) and the residual is extracted, as highlighted in Figure S6df. The temperature change was measured for each system using the same time points as in Figure S6.
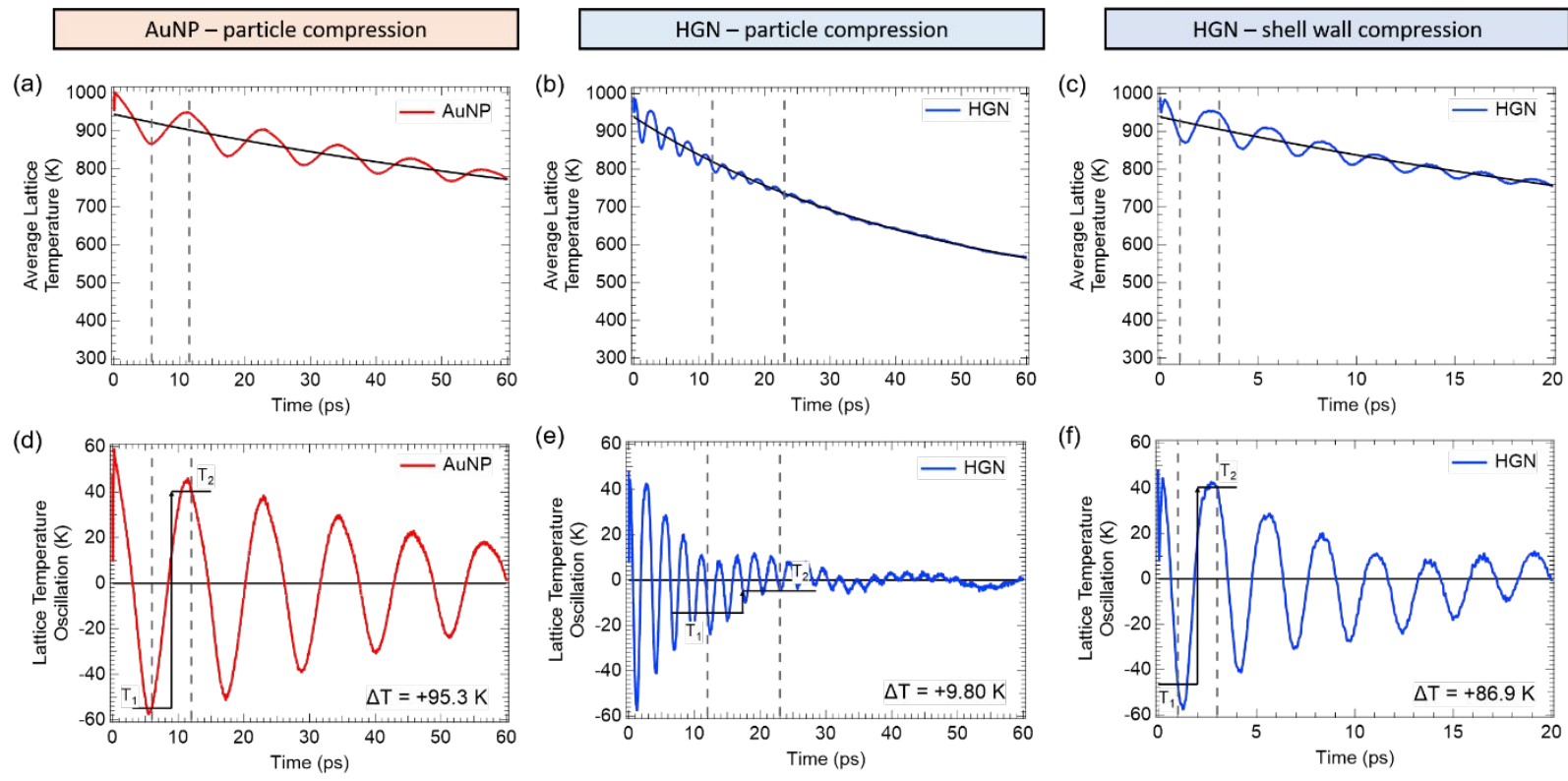

Figure S7. MD Simulation of Average Lattice Temperatures. Decay of average lattice temperature after heating to $1000 \mathrm{~K}$ for (a) AuNP and (b,c) HGN over 0-60 ps or 0-20 ps. Exponential decay trendlines are provided in black. (d-f) Corresponding average lattice temperature oscillations extracted by removing the bi-exponential decays. Dashed lines are provided to note the time points closest to the first observable compression: 6 ps and 12 ps for AuNP, 12 ps and 23 ps for HGN (low frequency oscillation), and 1 ps and 3 ps for the HGN shell wall (high frequency oscillation).

Our MD simulations show a temperature increase of $95.3 \mathrm{~K}$ for AuNP compression, in excellent agreement with the analytical estimate (95 K). For HGN compression, our MD simulations show a temperature increase of $9.80 \mathrm{~K}$, significantly lower than the temperature extracted from the analytical estimate $(34 \mathrm{~K})$. This difference may be due to the greater deviation from the isentropic 
regime, as mentioned. Finally, we estimate the temperature change associated with the high frequency compression of the HGN shell wall to be $86.9 \mathrm{~K}$. Interestingly, this is again in good agreement with the temperature extracted from the analytical estimate $(82 \mathrm{~K})$. Because the high frequency shell wall compression occurs over a much shorter time period (1-3 ps) than the low frequency whole-particle compression (12-23 ps), a limited amount of heat is lost to the surroundings, and it may more closely approximate the isentropic regime. All values are tabulated in Table S1.

Table S1. Analytical estimates of the temperature increase of the gold lattice during compression using the Grüneisen model, for both AuNP and HGN structures after thermal excitation to 1000 K. $\Gamma$ is the Grüneisen parameter for gold. The values of $t_{1}, t_{2}, r_{1}, r_{2}$, and $T_{1}$ were taken from the first compression cycle in MD simulation. Grüneisen $T_{2}$ represents the expected temperature increase of the gold lattice during isentropic compression.

\begin{tabular}{|c|c|c|c|c|c|c|c|c|c|c|c|}
\hline \multicolumn{4}{|c|}{ AuNP - particle compression } & \multicolumn{4}{|c|}{ HGN - particle compression } & \multicolumn{4}{|c|}{ HGN - particle shell wall compression } \\
\hline$\Gamma$ & 3 & & & $\Gamma$ & 3 & & & $\Gamma$ & 3 & & \\
\hline$t_{1}(p s)$ & 6 & $t_{2}(p s)$ & 12 & $t_{1}(p s)$ & 12 & $t_{2}(p s)$ & 23 & $t_{1}(p s)$ & 1 & $t_{2}(p s)$ & 3 \\
\hline \multirow{2}{*}{$r_{1}(n m)$} & \multirow{2}{*}{15.647} & \multirow{2}{*}{$r_{2}(n m)$} & \multirow{2}{*}{15.468} & $r_{01}(n m)$ & 15.011 & $r_{02}(n m)$ & 14.912 & $r_{01}(n m)$ & 14.9355 & $r_{02}(n m)$ & 14.900 \\
\hline & & & & $r_{i 1}(n m)$ & 11.016 & $r_{i 2}(n m)$ & 10.911 & $r_{i 1}(n m)$ & 10.818 & $r_{i 2}(n m)$ & 10.920 \\
\hline$V_{1}\left(\mathrm{~nm}^{3}\right)$ & 16047 & $V_{2}\left(n^{3}\right)$ & 15502 & $V_{1}\left(\mathrm{~nm}^{3}\right)$ & 8568 & $V_{2}\left(n^{3}\right)$ & 8450 & $V_{1}\left(n^{3}\right)$ & 8652 & $V_{2}\left(n^{3}\right)$ & 8402 \\
\hline $\mathrm{T}_{1}(\mathrm{~K})$ & 866 & $\mathrm{~T}_{2}(\mathrm{~K})$ & $?$ & $\mathrm{~T}_{1}(\mathrm{~K})$ & 804 & $\mathrm{~T}_{2}(\mathrm{~K})$ & $?$ & $\mathrm{~T}_{1}(\mathrm{~K})$ & 884 & $\mathrm{~T}_{2}(\mathrm{~K})$ & $?$ \\
\hline \multicolumn{3}{|c|}{ Grüneisen T2 $(K)$} & 961 & \multicolumn{3}{|c|}{ Grüneisen T2 (K) } & 838 & \multicolumn{3}{|c|}{ Grüneisen T2 (K) } & 966 \\
\hline \multicolumn{3}{|c|}{ Grüneisen $\Delta \mathrm{T}(\mathrm{K})$} & 95 & \multicolumn{3}{|c|}{ Grüneisen $\Delta \mathrm{T}(\mathrm{K})$} & 34 & \multicolumn{3}{|c|}{ Grüneisen $\Delta \mathrm{T}(\mathrm{K})$} & 82 \\
\hline \multicolumn{3}{|c|}{ MD Simulation $\Delta \mathrm{T}(\mathrm{K})$} & 95.3 & \multicolumn{3}{|c|}{ MD Simulation $\Delta \mathrm{T}(\mathrm{K})$} & 9.80 & \multicolumn{3}{|c|}{ MD Simulation $\Delta \mathrm{T}(\mathrm{K})$} & 86.9 \\
\hline
\end{tabular}

The Grüneisen Equation of State has been used to support the average temperature oscillations in the lattice. It is generally assumed that metal NPs have uniform temperature distributions due to the small length scales and relatively high thermal conductivities, but this assumption is not valid on ultrafast timescales, as we show in the manuscript (see Figure $4 \mathrm{~b}, \mathrm{e}$ ). As the hot nanoparticle transfers thermal energy to the water, thermal gradients in the surrounding water (and NP lattice) will develop over time causing a change in the spatial distribution of water (and metal) dielectric values. The result is that the oscillations near the particle surface are much lower in intensity than those of the average lattice. Additionally, the oscillations near the particle center are higher in intensity than those of the average lattice. These larger oscillations can be explained by constructive interference of related pressure waves due to spherical symmetry. 


\section{References}

1. Yokoo, M.; Kawai, N.; Nakamura, K. G.; Kondo, K.; Tange, Y.; Tsuchiya, T. Ultrahigh-Pressure Scales for Gold and Platinum at Pressures up to 550 GPa. Phys. Rev. B 2009, 80, 104114, 1-9.

2. Pamato, M. G.; Wood, I. G.; Dobson, D. P.; Hunt, S. A.; Vočadlo, L. The Thermal Expansion of Gold: Point Defect Concentrations and Pre-Melting in a Face-Centred Cubic Metal. J. Appl. Cryst. 2018, 51, 470-480.

3. Gupta, O. P.; Kharoo, H. L. Analysis of the Grüneisen Parameters of Some FCC Metals by an Improved Elastic Force Model. J. Chem. Phys. 1981, 74, 3577-3583.

4. Prut, V. V. Modeling and Simulation of Gruneisen Parameter for a Shock Wave. Russian Physics Journal 2019, 62, 2, 339-345. 